\title{
Empirical Patterns of High-Energy Particle Dynamics of the Van Allen Radiation Belts
}

\author{
Yuhan Liu \\ United World College South East Asia, Singapore \\ Email: serenaliu0510@gmail.com
}

How to cite this paper: Liu, Y.H. (2020) Empirical Patterns of High-Energy Particle Dynamics of the Van Allen Radiation Belts. Advances in Aerospace Science and Technology, 5, 45-57. https://doi.org/10.4236/aast.2020.52003

Received: March 1, 2020

Accepted: June 20, 2020

Published: June 23, 2020

Copyright (c) 2020 by author(s) and Scientific Research Publishing Inc. This work is licensed under the Creative Commons Attribution International License (CC BY 4.0).

http://creativecommons.org/licenses/by/4.0/

(c) (i) Open Access

\begin{abstract}
The Van Allen radiation belts consist of megaelectron volt particles trapped in the Earth's magnetic field mainly with protons at low altitudes and electrons at high altitudes. These concentric, donut-shaped radiation belts are constantly changing due to a variety of physical processes caused by specific types of heliospheric structure. The radiation poses risks for astronauts and spacecraft systems. The understanding of these processes that operate across the universe can help with designs of future space missions as well as the understanding of the universe. The data gathered from the last three months of the Radiation Belt Storm Probes mission (RBSP mission) is examined for patterns and trends in the dynamics of the radiation belts and their relationships with solar activities. From the data, it is shown that the patterns of the high-energy particle dynamics in the belts follow the changes in solar activity. When the Sun's magnetic field weakens, cosmic rays from deep space are able to carry an increased flux of energetic particles into the solar system. The outer belt exhibits a pattern of electron energization during cycles lasting an average of 27 days, corresponding to the time taken for one solar rotation. The sudden outward shift of the belts on July 19th, 2019 was most likely caused by the shutdown of one of the two Van Allen probes, as the relative position of the probe to the belts changed. Two solar wind streams that arrived from August 27th to the 28th caused the "dropout" event on August 28 th, as the contact between the solar wind streams and magnetosphere pushed the magnetosphere's boundary and enabled the particles outside the shifted boundary to escape into space. A positive polarity coronal hole high speed stream (CH HSS) contributed to the intense September electron re-energization process in the radiation belts starting on September 1st.
\end{abstract}

\section{Keywords}

Van Allen Radiation Belt, Relativistic Electron Dynamics, Geomagnetic Storms, Solar Cycles 


\section{Introduction}

As the first major scientific discovery of the space age, the Van Allen radiation belts surrounding Earth acts as a shield to protect the planet from harmful solar winds. The Van Allen radiation belts are composed of energetically charged particles captured by the Earth's magnetic field. The charged particles carried by solar winds enter Earth's magnetosphere and experience acceleration or neutron decay. The radiation belts also pose a serious challenge for space travel, as astronauts leaving Earth's orbit have to go across the radiation belts, and the high speed subatomic particles can damage DNA and increase the risk of cancer and other diseases. They can also damage spacecraft systems, cause power grid failures and disrupt GPS communications.

The two spatial populations of the Van Allen radiation belts are the inner and outer zones. The inner belt-located about 1000 - 8000 miles away from Earth's surface-consists of high-energy electrons and energetic protons produced by cosmic rays interacting with the atmosphere. The outer belt-located between 12,000 - 25,000 miles away from the Earth's surface-consists of mostly megaelectron volt electrons. The changes of the radiation belts depend on the relativistic electron dynamics in the magnetosphere, which is yet to be fully resolved and predicted. While the inner belt is mostly stable in intensity levels over the years, the outer belt waxes and wanes, constantly changing in intensity. As the inner belt is nearer to Earth, the particles are held on with a stronger magnetic force with protons that are restricted to the inner belt. Major disturbances in the magnetosphere caused by high energy exchanges (e.g. sunspots, solar flares and coronal mass ejections) from the solar wind can cause geomagnetic storms, which can result in changes in the radiation belts and potentially damage satellites [1].

There are three main physical processes that contribute to the changes in particle flux in the radiation belts: Precipitation, Transportation of particles and change in energy distribution. Precipitation is the change in pitch angle caused by wave particle interaction that scatters the particles and forces them down into the planet's atmosphere. The transportation of particles results in changes in the radiation belts as the particles are constituents of the belts. Change in energy distribution changes the particle flux in the radiation belts as spatial activities can energize or de-energize particles [2].

Radiation belt particles move quickly, circling the belt's magnetic field in a few milliseconds while bouncing back and forth as they are trapped in the belt's magnetic field lines. The motion of the particles drift along the field line in an eastward flow around the Earth is known as radial diffusion. All motions combine to become what is known as the adiabatic invariants. During geomagnetic storms, the drift paths expand outward, causing the radiation belts fluxes to undergo a repeating decrease and increase. Many other processes also contribute to changes in the radiation belts. For example, the cosmic ray albedo neutron decay (CRAND) continuously adds ionized particles (protons) into the inner belt [3].

The RBSP mission aims to enhance the understanding of the Sun's influence 
on Earth and near-Earth space by investigating Earth's radiation belts on various scales and time. The Van Allen Probes used in the RBSP mission fly through the radiation belts at about $3220 \mathrm{kmh}$, passing through varying and harsh conditions with sets of instruments to measure charged particle populations, fields, and waves in the inner magnetosphere. As data observed from one spacecraft can be unreliable because of the uncertainty of travelling disturbances, the twin probes confirm the accuracy of the data. The Relativistic Electron-Proton Telescope (REPT) on board of the probes measures electron population from 1 to $30 \mathrm{MeV}$ throughout the orbit. The probes travel in an elliptical orbit approximately in the equatorial plane with perigee about 1.2 Earth radii and apogee about 6 Earth radii. After seven years of operation, one of the Van Allen probes shut down on July 18th 2019. This article examines the long term trends and patterns of the radiation belts and tries to prove the correlation between solar activities and the energetic particle dynamics in the Van Allen Radiation Belts. The most recent data from the RBSP mission is visualized and analysed by plotting the Mcilwain $\mathrm{L}$ parameter ${ }^{1}$ of the satellite orbit as a function of time with the electron log-flux variation expressed with color scale in separate panels for the different energy channels of electrons to show the trend of high energy electron decay, refill and acceleration process.

\section{Literature Review}

The two-belt structure for electrons is the result of wave-particle interactions. It was found in 2013 that the wave-particle interactions also accelerate the electrons trapped in the belts, resulting in large increases in electron flux. Megaelectron volt $(\mathrm{MeV})$ ions are trapped in the inner belt of the two-belt structure of the Van Allen Radiation Belts due to their larger gyroradii-the radius of the circular motion of a charged particle in the presence of a uniform magnetic field. The inner belt electron population is periodically refreshed by the transport of electrons from the outer belt. The decay of low thermal energy neutrons-a majority of albedo neutrons produced by cosmic rays striking the upper atmosphere-contributes to energetic electrons in the inner belt.

In August 2012, the twin Van Allen Probes (later renamed Radiation Belt Storm Probes (RBSP)) were launched into a $\sim 600 \times 33,000-\mathrm{km}$ orbit with an inclination of $\sim 10^{\circ}$. The probes provided detailed on site measurements during Solar Cycle 24. The twin probes travel in nearly identical orbits with variable separation, a $\sim 9 \mathrm{hr}$ orbital period with 19-month return cadence to the same magnetic local time [1].

The complexity and simultaneity of processes happening in the radiation belts makes them especially hard to study. Different processes are often driven by the same external factors and the speed at which these processes are happening further complicates the research.

${ }^{1}$ The Mcilwain L parameter describes a particular set of planetary magnetic field lines with L-value describes the magnetic field lines crossing Earth's magnetic equator at the L-value of Earth-radii. 
The Geospace exploration project ERG mission continues its onsite observations in the radiation belts [4]. There is a negative correlation between the magnitude of geospace storms and changes in the flux of relativistic electrons. Likewise, with data from 2015 to the end of 2018, the decrease in solar activity has led to an increased flux of energetic particles [5]. Researchers are trying to figure out which of the two mechanisms-external source process via quasi-adiabatic acceleration or radial diffusion-causes the large flux enhancement of the outer radiation belt electrons.

During geomagnetic storms, the inner magnetospheric field inflates and results in the expansion of the drift paths of particles. This influence is known as the "Dst effect" as the field inflation tracks the disturbance storm-time index, Dst. This process causes the radiation belt fluxes to undergo a predictable and repeatable fluctuation (decrease and the increase) during geomagnetic storms. A variety of other processes such as SEP injections, CRAND ion trapping, radial diffusion, etc are also at work. Some of these processes enhance the radiation belts while other loss mechanisms suppress the belts' intensity. The data shows roughly half of geomagnetic storms result in a flux decrease while the other half evenly splits between a flux decrease or roughly no change from the prestorm flux intensity [6].

An important source of radiation belt enhancement happens when a galactic cosmic ray strikes the upper atmosphere, creating a neutron which later decays into a proton before it leaves the magnetosphere. Known as the cosmic ray albedo neutron decay (CRAND), this process provides a small but constant addition to the inner zone ions. Other sources contributing to radiation belt enhancement include the trapping of solar energetic particles from solar flares which often results in a sudden increase of the ion content within the radiation belts. The radiation belt ions can escape through magnetopause, similarly, electrons are subject to the Dst effect and losses at magnetopause. Additionally, plasma waves-electric and magnetic field vibrations that interact with the charged particles-is a main contributor in changing the distribution of radiation belt electrons on timescales of a few hours to days [7].

Radiation belt dropout is one of the most dramatic variations in the radiation belt, with the electron flux observed to drop by several orders of magnitude in a span of a few hours. Three distinct radiation belt dropout events are investigated by Xiang et al. (2017) [8] for a better understanding of the dominant loss mechanisms of radiation belt electrons. The findings suggest that these radiation belt dropout events can be categorized into three classes according to dominant loss mechanisms: magnetopause shadowing dominant, EMIC wave scattering dominant, and combination of both mechanisms.

\section{Data}

Using data from the RBSP database from the past 3 months, the relationship between electron flux and L-values in different times is plotted. The data is visualized using several packages on Python, such as NumPy and Matplotlib. First, 
the data over each month is downloaded and imported into the program, then the variables-Epoch, FESA, Energy and L-are set up with the correct time stamp. Epoch and $\mathrm{L}$ are one-dimensional variables, representing time and $\mathrm{L}$ value. FESA is a two-dimensional variable, representing electron distribution at different times and energy levels. A loop is then constructed, concatenating the variables of the same type from each day before plotting and combining all data from each variable from each day of the month.

\section{Analysis}

From the three-month plot in Figure 1, it is shown that at the beginning of July the radiation belts were at the end stage of a decay period, with the inner and outer belts both having an electron flux of about $10^{4} \mathrm{~cm}^{2}-\mathrm{s}-\mathrm{sr}-\mathrm{MeV}$ with a stable slot region (formed as a result of losses due to interactions between electrons and certain electromagnetic waves) at L 3. Beginning on July 11th, a rapid increase of electron flux in the outer belt occurred, suggesting the re-energization of the outer belt. The energization first appeared in the highest altitude of the radiation belt at $\mathrm{L} \sim 6$, with electron flux of about $10^{7} \mathrm{~cm}^{2}-\mathrm{s}-\mathrm{sr}-\mathrm{MeV}$. The energization process continued for about 7 days, resulting in a uniform increase in electron flux in region $\mathrm{L} \sim 4$ to $\mathrm{L} \sim 6$. The electrons then decayed slowly until the next re-energization on August 6th. From the long term data analysis, the electron flux of the inner radiation belt remained mostly constant throughout the months, except for the observed shifted position in the radiation belts on July 18th. The outer belt exhibits a pattern of electron energization during cycles lasting an average of 27 days. As annotated in Figure 1, there is a notable shift of one L-value in the position of the inner and outer belt and the slot regions at the end of July away from Earth. The shifted position remained for about nine days before the belts underwent a shift of about half an L-value closer to the Earth. At this point, a weak "storage ring" [9] appeared after the observed inward shift and remained for the month of August at $\mathrm{L} \sim 3$. It is notable that the energization process in August persisted much longer in time than that of July with a sharp decrease in electron flux at the end of the month. At the start of September, the radiation belts then returned to their usual two-belt structure as the outer belt re-energized. Compared to July, where the electron flux increased only from $\mathrm{L} \sim 4.5$ to $\mathrm{L} \sim 6$ initially, the energization process in August and September is more intense, with an increase in electron flux throughout the region from $\mathrm{L} \sim 4$ to $\mathrm{L} \sim 6$ from the beginning.

Figures 2-5 demonstrate that electrons of higher energy channels scatter around in the inner radiation belt, and that the outer belt consists of electrons of lower energy channels. The total electron flux of particles with lower energy is much higher than those of higher energy. Particles with lower energy are also more prominent throughout the belts, occupying a majority of the outer belt area while particles at a higher energy channel of $7.7 \mathrm{MeV}$ only exist and are concentrated in the inner belt at $\mathrm{L} \sim 2$ and $\mathrm{L} \sim 1$. Comparing the three individual 
graphs of each month, it is shown that the time period of electron energization and decay differs. The electrons were energized for about 12 days before decaying rapidly for the next 13 days in July. The energization period in August was shorter with a long decay time and lower decay rate followed by a sudden increase in decay rate (a "dropout" event) in the last two days of August. In September, the radiation belts experienced electron energization for about nine days followed by a constant and gradual decay for the rest of the month.

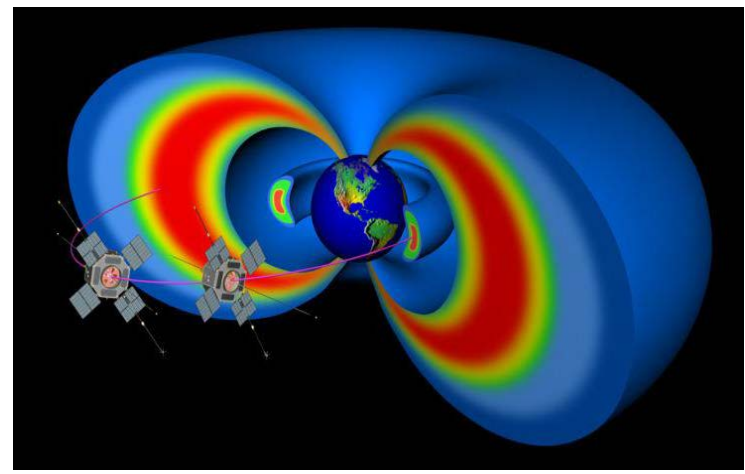

Figure 1. Artist's rendering showing the twin probes studying the Van Allen Radiation Belts. The figure was produced by Johns Hopkins University Applied Physics Laboratory.

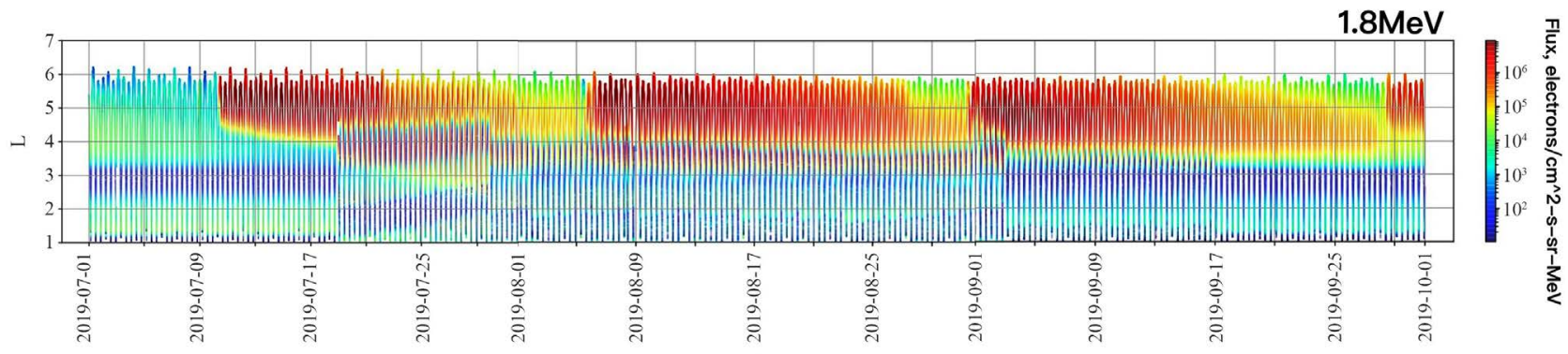

Time

(a)

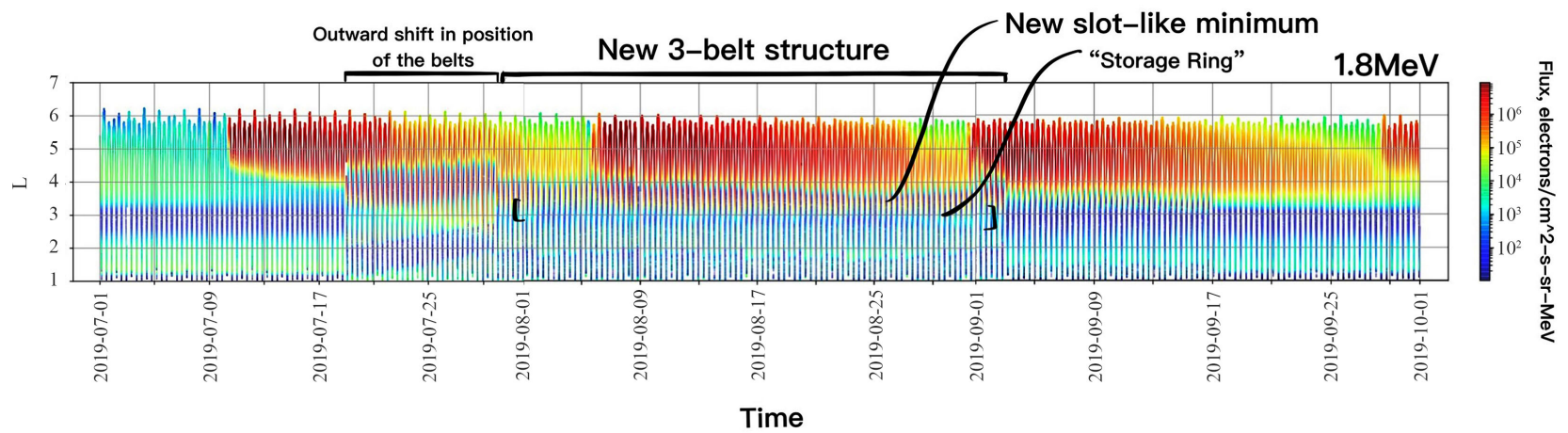

(b)

Figure 2. (a) Data visualization over the three-month period from July to September of 2019, showing the long term trend of energetic electron dynamics in the energy channel of $1.8 \mathrm{MeV}$. L-values are plotted on the $\mathrm{y}$-axis with time as the $\mathrm{x}$-axis with flux in a color coded logarithmic scale. The data from the $1.8 \mathrm{MeV}$ energy channel is plotted. (b) Annotated plot for Figure 1, showing notable changes and features in the three-month data visualization. 

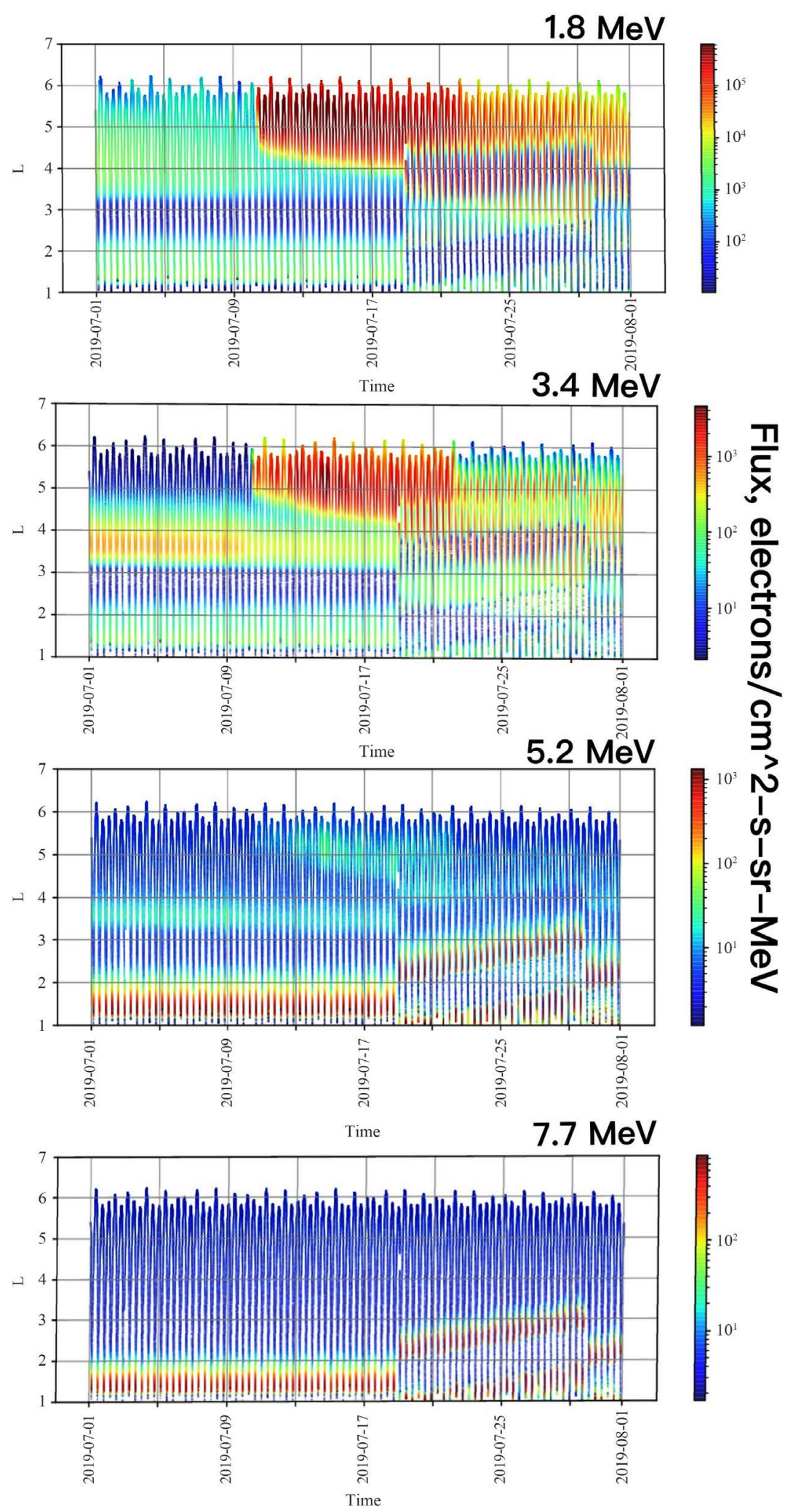

Figure 3. Energetic electron data from the RBSP satellites gathered from the month of July 2019 is visualized by plotting the $\mathrm{L}$ values on the $\mathrm{y}$-axis and time on the $\mathrm{x}$-axis with electron flux in a color-coded logarithmic scale. Four discrete energy channels of the $\mathrm{MeV}$ particles are plotted. 

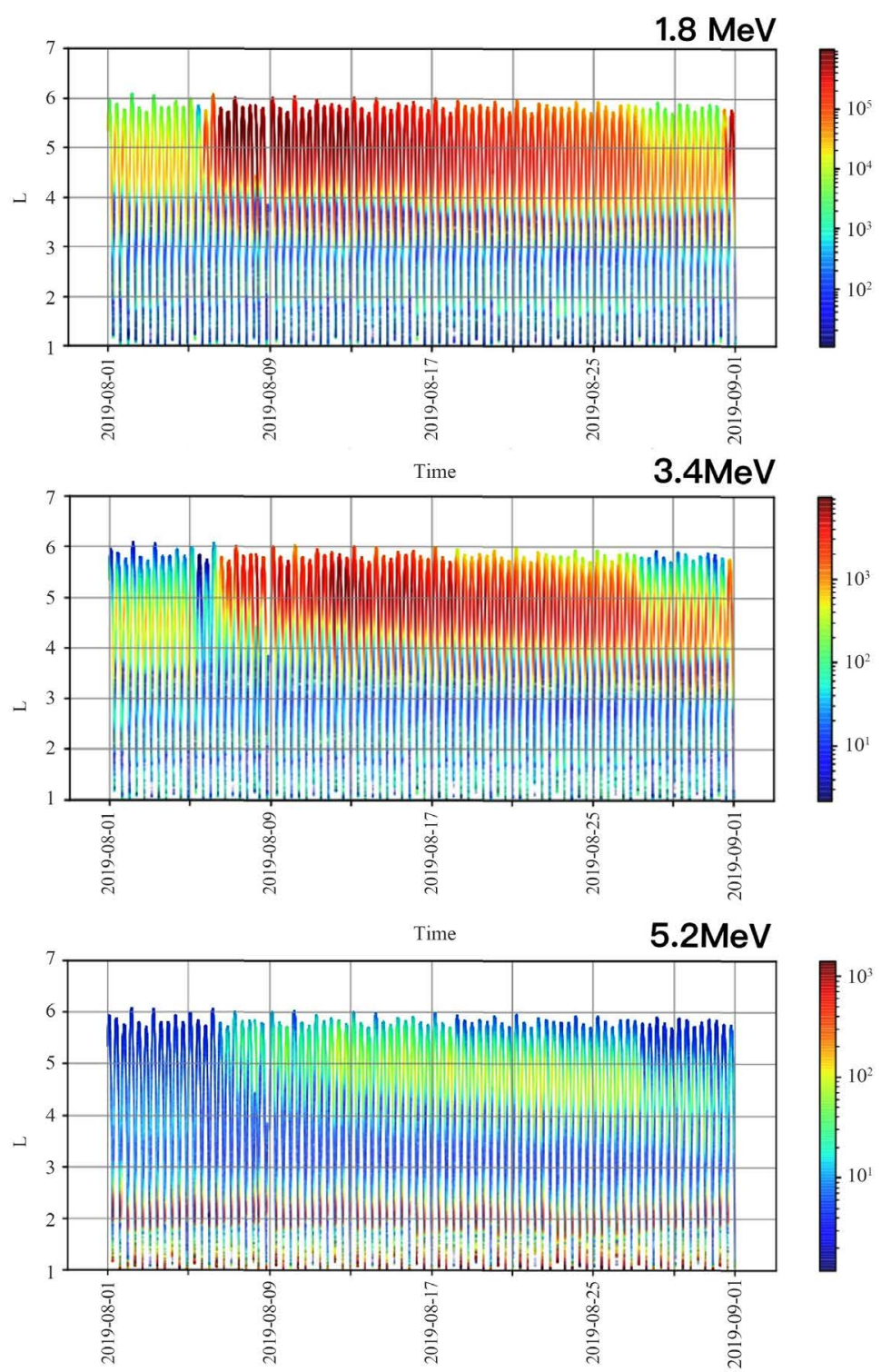

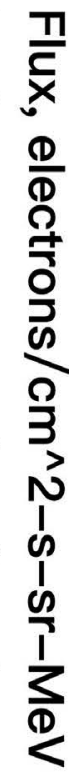

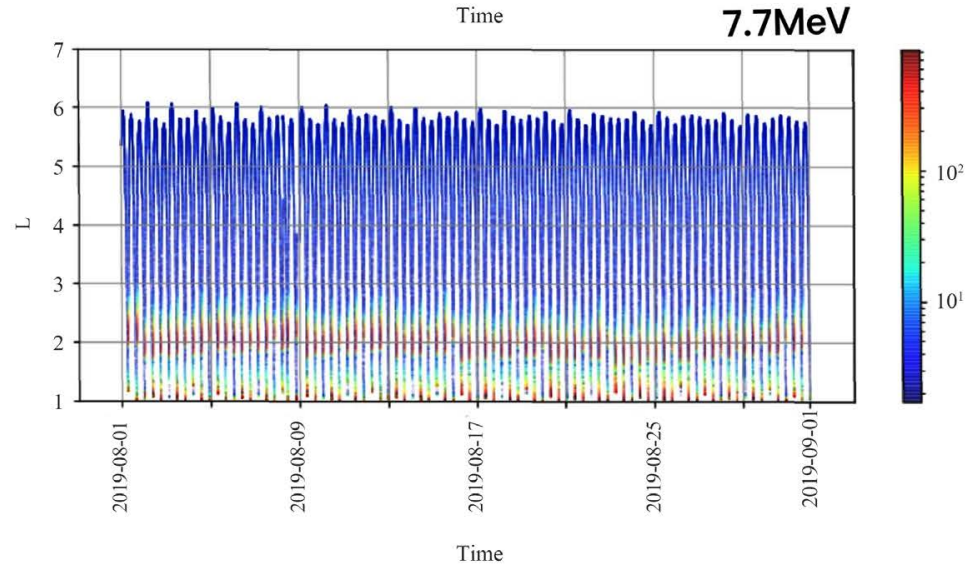

Figure 4. Energetic electron data from the RBSP satellites gathered from the month of August 2019 is visualized by plotting the L values on the $y$-axis and time on the $\mathrm{x}$-axis with electron flux in a color-coded logarithmic scale. Four discrete energy channels of the $\mathrm{MeV}$ particles are plotted. 

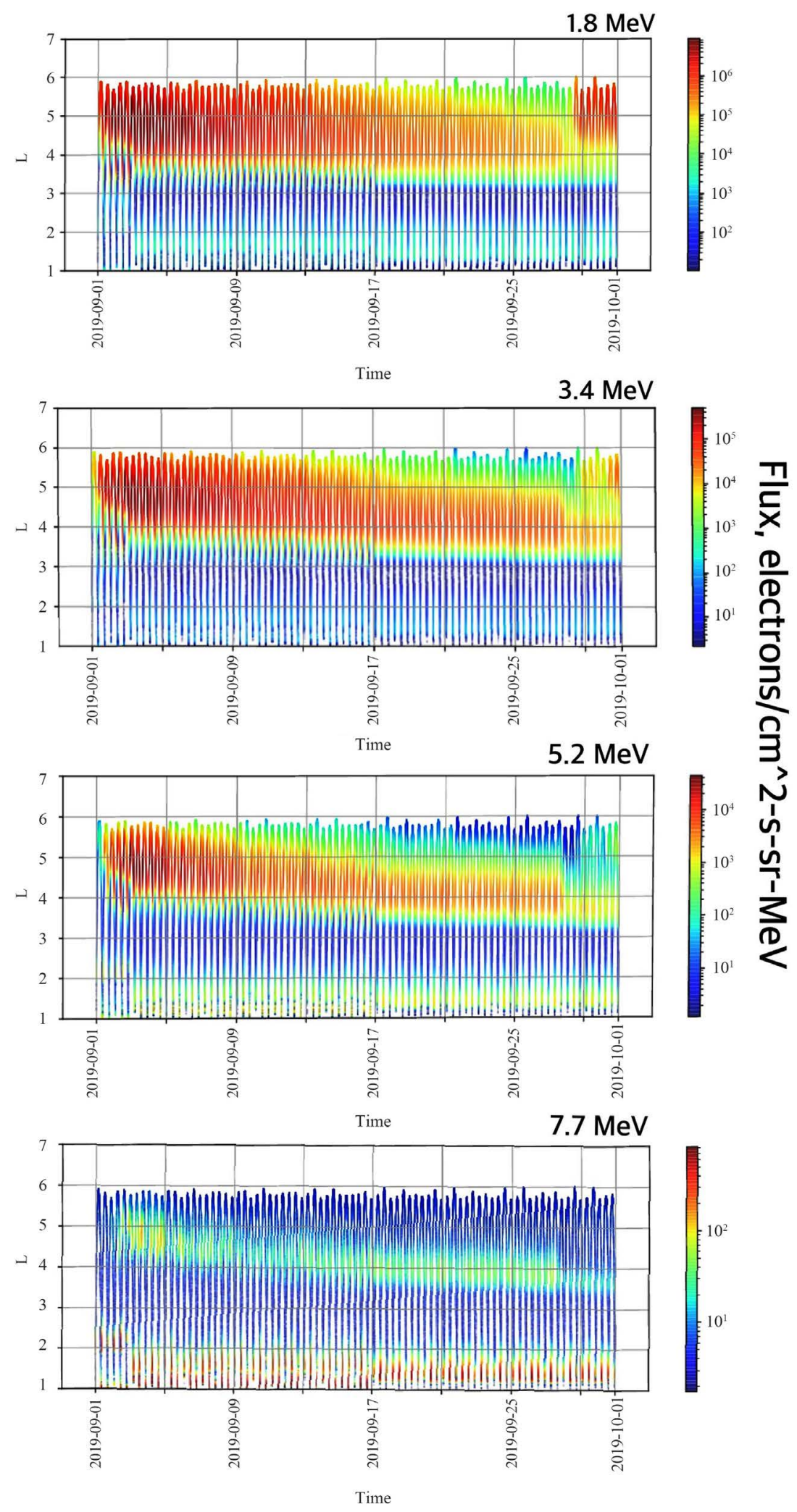

Figure 5. Energetic electron data from the RBSP satellites gathered from the month of September 2019 is visualized by plotting the $L$ values on the $y$-axis and time on the $\mathrm{x}$-axis with electron flux in a color-coded logarithmic scale. Four discrete energy channels of the $\mathrm{MeV}$ particles are plotted. 


\section{Results}

One of the deepest Solar Minima of the 21st century was underway in the year 2019. As the Sun's magnetic field weakened, the overall decrease in solar activity has led to an increased flux of energetic particles as cosmic rays from deep space flood into the solar system. Measurements from weather balloons show that cosmic rays are intensifying with an increase of more than $18 \%$ since 2015 [10], demonstrating the effects of a solar minimum. As the particles are energized through wave-particles interactions, high energy exchanges such as solar wind and coronal mass ejections which eject particles and plasma towards Earth can cause geomagnetic storms, creating wave-particles interactions needed to energize the particles. Particles from solar activities such as sunspots, solar flares and coronal mass ejections that happen to be ejected towards Earth can be trapped in Earth's magnetic field, contributing to the increase of the electron flux in the radiation belts (Figure 6).

The particle re-energization and decay repeat in a 27-day cycle, corresponding to the period of solar rotation. As solar rotation has a significant impact on coronal expansion, wind speed, and the orientation of the wind, different phases of the solar rotation affect the generation of shock waves as well as other solar activities. During declining phases of the solar cycle, regions with slow wind and fast wind would both face Earth at different times. In this case, the gas in between becomes compressed as fast wind overtakes the slow wind, forming a shock wave which energizes the particle as it approaches the radiation belts. The 27-day cycle of the particle dynamics in the radiation belts shows the direct relation between solar activities and the changes in the radiation belts. These solar activities can energize particles and inject them into the radiation belts. The outward shift of the radiation belt away from Earth on July 19th was most likely caused by the shutdown of one of the two Van Allen probes on the same day, as the absence of the second probe changed the relative position where the data is collected. The following data after July 19th was shown with fluctuations and unclear bands, suggesting travelling disturbances from the one probe (Figure 7).

The "dropout" event on August 28th-when there was a sudden loss of electrons in the outer belt-can be a result of two solar wind streams which arrived from August 27th to 28th [11]. When the solar wind comes in contact with the magnetosphere, the magnetosphere's boundary is pushed within the radiation belts. The particles from the radiation belts beyond the shifted boundary (the magnetopause) have a greater chance of escaping into space. The change of the shape of the radiation belts interferes with the particles' typical drift around the center mass and electrons are forced outwards, away from Earth. The solar wind can also enable plasma within the magnetosphere to generate waves of energy and scatter electrons (precipitation). The electrons can be forced down into the atmosphere as they spin and bounce because of the extra energy, thus the sudden decrease in electrons. The dropout also includes the depletion of the storage ring at the end of August, returning the radiation belts to the usual two-belt structure. 


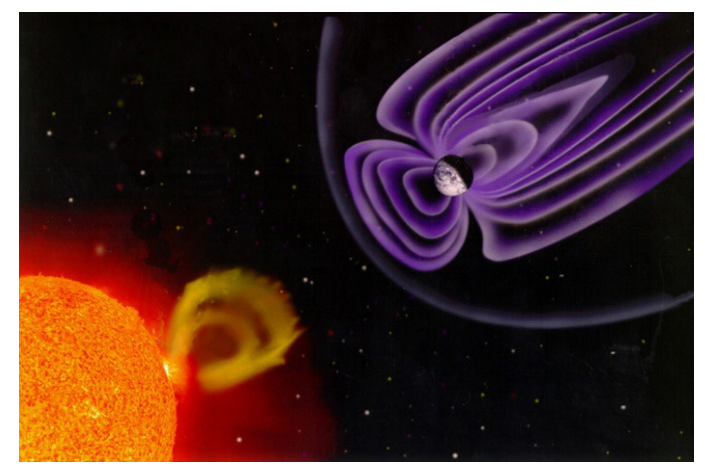

Figure 6. Solar activities such as coronal mass ejections can cause geomagnetic storms on Earth. Located in the Earth's magnetosphere, the Van Allen Radiation Belts changes responding to solar activities. This figure is adapted from NASA.

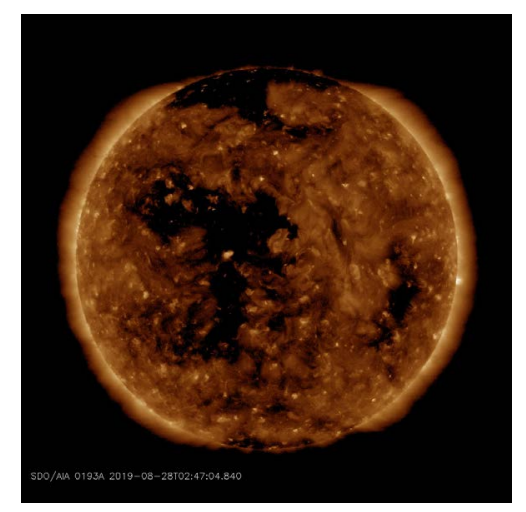

Figure 7. The figure shows the coronal hole (dark spots on the Sun) facing Earth on 28 August, solar wind flowing from this large coronal hole reached Earth on August 27 to 18 and September 1, first causing the dropout event then initiating the particle energization process. Figure adapted from SDO/AIA.

At the beginning of September, geomagnetic storms were associated with a positive polarity coronal hole high speed stream (CH HSS) [12]. As the magnetic field lines of a coronal hole stay open and stretched out into space, where solar wind can escape at high speeds without the hindrance of the magnetic force, often causing geomagnetic storms on Earth. Solar wind speed reached in excess of $800 \mathrm{~km} / \mathrm{s}$ with the coronal hole. On September 1st, a stream of high-speed solar wind lashed through Earth's magnetic field, travelling at speeds greater than 700 $\mathrm{km} / \mathrm{s}$. The September electron re-energization was the most intense in the three-month period. There was a peak in the KP index ${ }^{2}$ showing directly how high-energy dynamics in the radiation belts affects geomagnetic activity on Earth. During this time, there was a sharp decrease in the DST index ${ }^{3}$ as the storm current intensified in Earth's magnetic field [13]. As mentioned above, solar wind and geomagnetic storms can change the belts' structure. After the geomagnetic storm in the beginning of September, the radiation belts shifted backwards, returning to the position before the outward shift of July.

${ }^{2} \mathrm{KP}$ index: global geomagnetic activity index from magnetometers around the world each calibrated according to its latitude.

${ }^{3}$ DST index: a measure of geomagnetic activity used to assess the severity of magnetic storms. 


\section{Summary}

The final 3-month data before the end of the RBSP mission was visualized and analysed for trends and relationships between solar activities and particle dynamics in the radiation belts. The radiation belts re-energize every solar rotation, and exchange energy and particles from the Sun before decaying. While some solar activities energize the radiation belts, others de-energize it. Particles can escape the belts or scatter and force particles to precipitate into Earth's atmosphere as the shape of the radiation belts changes. From the data, it is shown that the patterns of the high-energy particle dynamics in the belts follow the changes in solar activity. When the Sun's magnetic field weakens, cosmic rays from deep space are able to carry an increased flux of energetic particles into the solar system. The outer belt exhibits a pattern of electron energization during cycles lasting an average of 27 days, corresponding to the time taken for one solar rotation. The sudden shift in position of the belts in July possibly resulted from inaccuracies due to travel disturbances caused by the shutdown of one of the twin probes. Two solar wind streams that arrived from August 27th to the 28th caused the "dropout" event on August 28th, as the contact between the solar wind streams and magnetosphere pushed the magnetosphere's boundary and enabled the particles outside the shifted boundary to escape into space. A positive polarity coronal hole high speed stream ( $\mathrm{CH}$ HSS) contributed to the intense September electron re-energization process in the radiation belts starting on September 1 st.

The simultaneity of the dynamic interplay of processes that contributes to the variability of the belts makes it difficult for scientists to pinpoint and understand the effects of each process and the fundamental physics of the Van Allen Radiation Belts. Although the general trends of the particle dynamics of the belts can be explained, there still remain open questions and work to do. Many of the challenges faced involve the modelling of radiation belts and incorporating radiation belt physics into other models such as the global geospace model to enhance the forecast of space weather. The research on the underlying physics of the Van Allen Radiation Belts could further the understanding of energetic particle dynamics on other astronomical bodies such as magnetized planets within and outside the solar system as well as astrophysical and laboratory plasmas. The understanding of the radiation belts can also help enhance technological systems to counter the impacts of space weather. Although the RBSP mission is coming to an end, future missions including the NASA Living With a Star Radiation Belt Storm Probes (LWS RBSP) and the Canadian Outer Radiation Belt Injection, Transport, Acceleration and Loss Satellite (ORBITALS) missions, ERG (Arase) satellite continue to explore the radiation belts. These future missions aim to understand the dominant processes causing flux enhancement of relativistic electrons, the effects of the wave-particle interactions and the dominant loss processes for the relativistic electrons of the outer radiation belt and other open questions [4]. 


\section{Conflicts of Interest}

The author declares no conflicts of interest regarding the publication of this paper.

\section{References}

[1] Li, W. and Hudson, M. (2019) Earth's Van Allen Radiation Belts: From Discovery to the Van Allen Probes Era. Journal of Geophysical Research: Space Physics, 124, 8319-8351. https://doi.org/10.1029/2018JA025940

[2] Liemohn, M. and Chan, A. (2007) Unraveling the Causes of Radiation Belt Enhancements. Eos, Transactions American Geophysical Union, 88, 425-426. https://doi.org/10.1029/2007EO420001

[3] North, G.R., Pyle, J. and Zhang, F.Q. (2015) Encyclopedia of Atmospheric Sciences. Elsevier, Amsterdam.

https://www.sciencedirect.com/referencework/9780123822253/encyclopedia-of-atm ospheric-sciences

[4] Sakaguchi, K., et al. (2015) Prediction of MeV Electron Fluxes throughout the Outer Radiation Belt Using Multivariate Autoregressive Models. Space Weather, 13, 853-867. https://doi.org/10.1002/2015SW001254

[5] Miyoshi, Y., et al. (2018) Geospace Exploration Project ERG. Earth, Planets and Space, 70, 4. https://doi.org/10.1186/s40623-018-0862-0

[6] Reeves, G. (1998) Relativistic Electrons and Magnetic Storms: 1992-1995. Geophysical Research Letters, 25, 1817-1820. https://doi.org/10.1029/98GL01398

[7] Big News from the Magnetosphere (2020). https://www.planetary.org/blogs/guest-blogs/2018/0605-big-news-from-the-magnet osphere.html

[8] Xiang, Z., et al. (2017) Understanding the Mechanisms of Radiation Belt Dropouts Observed by Van Allen Probes. Journal of Geophysical Research: Space Physics, 122, 9858-9879. https://doi.org/10.1002/2017JA024487

[9] rPlot (2020) Van Allen Probes Science Gateway. http://rbspgway.jhuapl.edu/rPlotTime?context

[10] Zeitlin, C., et al. (2019) Update on Galactic Cosmic Ray Integral Flux Measurements in Lunar Orbit with CRaTER. Space Weather, 17, 1011-1017. https://doi.org/10.1029/2019SW002223

[11] (2020) Swe.ssa.esa.int. http://swe.ssa.esa.int/TECEES/spweather/workshops/proceedings_w1/SESSION2/b uehler_radiation.pdf

[12] Spaceweather.com Time Machine (2020).

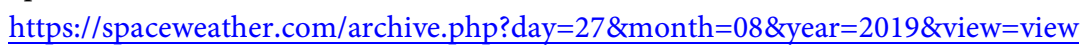

[13] Noaa.gov. (2020) Products and Data. NOAA/NWS Space Weather Prediction Center. https://www.swpc.noaa.gov/products-and-data 